\title{
Preoperative Optical Coherence Tomography Findings of Foveal-Splitting Rhegmatogenous Retinal Detachment
}

\author{
Valérie Manéa, ${ }^{a}$ Ismael Chehaibou ${ }^{a}$ Mathieu Lehmann ${ }^{b}$ Elise Philippakis ${ }^{a}$ \\ Pierre-Raphaël Rothschild ${ }^{b}$ Elodie Bousquet ${ }^{b}$ Ramin Tadayoni ${ }^{a}$

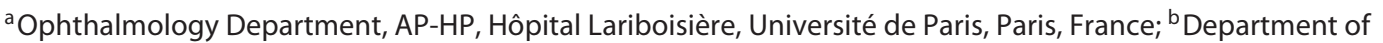 \\ Ophthalmology, Ophtalmopôle de Paris, AP-HP, Université Paris Descartes, Paris, France
}

\section{Keywords}

Optical coherence tomography $\cdot$ Rhegmatogenous retinal detachment · Fovea

\begin{abstract}
Purpose: To assess preoperative optical coherence tomography (OCT) findings of foveal-splitting retinal detachment (RD) and determine postoperative outcomes. Methods: Consecutive patients who underwent RD surgery over a 1 -year period were included. Patients diagnosed with a detachment extending to the edge of the fovea on fundus examination (i.e., macula-On/Off) underwent macular OCT scanning. Visual acuity (VA) after 1 year of macula-On/Off, macula-On, and macula-Off eyes was compared. Results: A total of 85 eyes were included, 8 of which had a macula-On/ Off RD. On preoperative OCT, all macula-On/Off RD eyes had foveal detachment extending beyond the foveal center over a median distance of $632 \mu \mathrm{m}$. Mean VA of the macula-On/Off eyes had improved from $20 / 160$ to $20 / 40$ at 1 year postoperatively $(p=0.035)$. The preoperative VA of macula-On/Off eyes was significantly better than macula-Off eyes $(p=0.032)$ and lower than macula-On eyes $(p=0.004)$. At 1 year, the VA of macula-On/Off eyes was no different from that of the mac-
\end{abstract}

ula-On eyes $(p=0.320)$, and tended to be better than that of the macula-Off eyes ( $p=0.062)$. Conclusion: Preoperative OCT revealed a shallow RD extending beyond the foveal center in eyes with clinical foveal-splitting RD. These eyes, termed macula-On/Off RD eyes, had a preoperative VA between macula-On and macula-Off eyes, while their final VA was close to those with macula-On RD.

(c) 2020 S. Karger AG, Basel

\section{Introduction}

An accurate assessment of retinal detachment (RD) boundaries is of clinical value. The status of the macula influences prognosis and may contribute to decisions about the urgency of surgery $[1,2]$. In fact, the detachment of the foveal center has been clearly identified as a negative prognostic factor for visual recovery after surgical repair [3]. In this regard, in cases of macula-On RD, surgery should be performed early, the main concern being the conversion into macula-Off $\mathrm{RD}$, for which the visual prognosis is poorer $[4,5]$. In cases of macula-Off $\mathrm{RD}$, the visual outcome is less dependent on early surgery and the surgical procedure can be delayed [6]. The preopera-

\footnotetext{
Valérie Mané

Service d'Ophtalmologie, Hôpital Lariboisière

2 rue Ambroise Paré

FR-75475 Paris Cedex 10 (France)

valerie.mane@yahoo.fr
} 

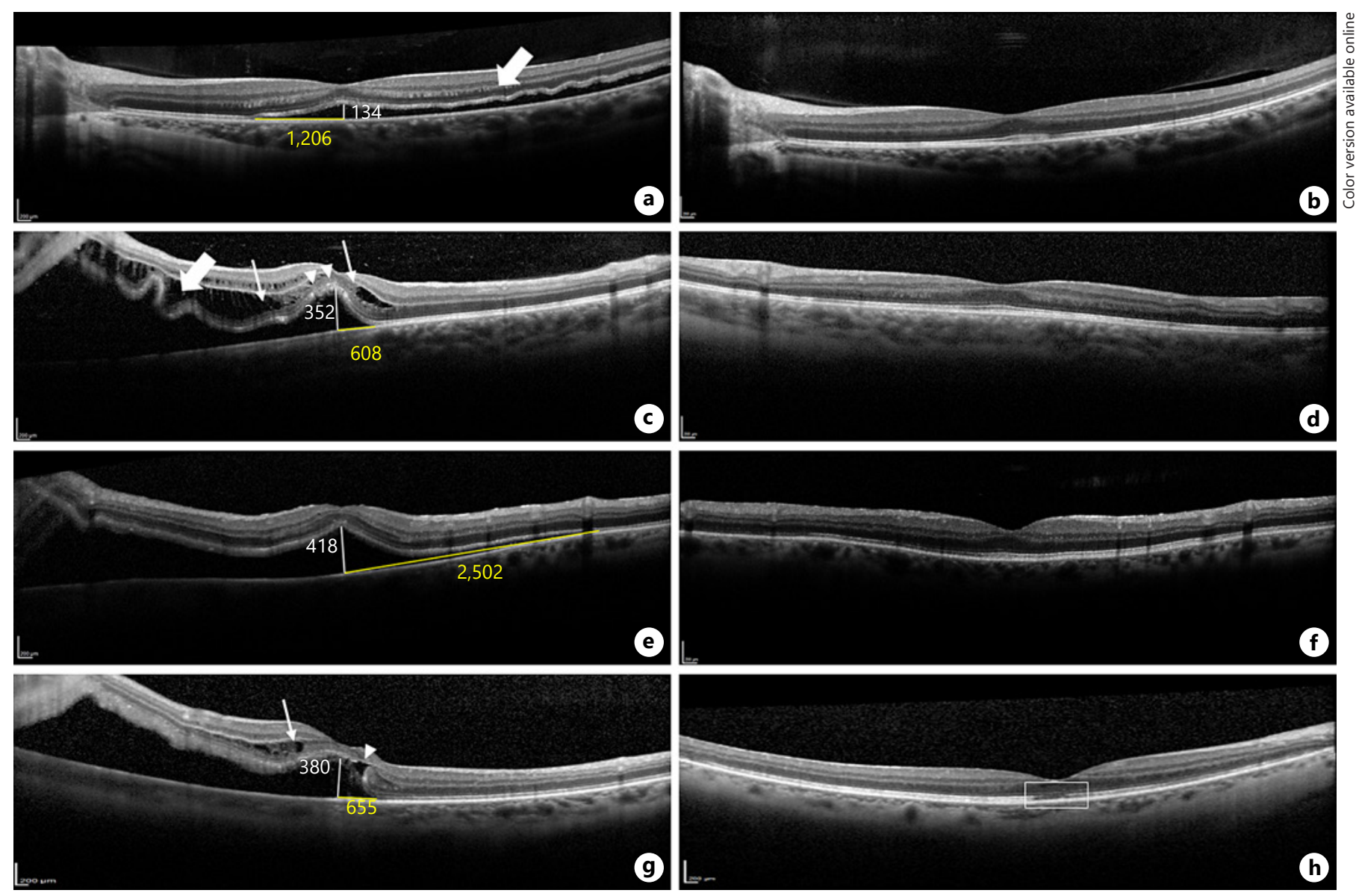

Fig. 1. Optical coherence tomography (OCT) scans of patients diagnosed with foveal-splitting retinal detachment (RD) on fundus examination at baseline and at the 1-year follow-up. At baseline (a, c, e, $\mathbf{g}$ ), a manual measurement of the distance between the foveal center and RD border (yellow line), and of RD height (white line) was performed $(\mu \mathrm{m})$. Intraretinal fluid $(\mathbf{c}, \mathbf{g}$, thin arrows), retinal

tive foveal status is currently determined from fundus examination and a clinician's experience.

Optical coherence tomography (OCT) imaging enables the detection of structural changes that are not always clinically evident on fundus examination $[7,8]$. In $\mathrm{RD}$, OCT has been used to diagnose persistent subretinal fluid that cannot be seen on fundus examination after surgery [9-12]. Similarly, on preoperative examination, OCT may be useful to detect a greater extent of RD and to better assess the involvement of the fovea. This study was designed with the aim of analyzing the presence and extent of infraclinical macular detachment using OCT in eyes diagnosed with foveal-splitting $\mathrm{RD}$ on clinical examination and compare the visual outcomes of these eyes with macula-On and macula-Off $\mathrm{RD}$ eyes.

corrugation (a, c, thick arrows), ellipsoid-zone and external limiting membrane (ELM) disruption (c, $\mathbf{g}$, arrowhead) was visible preoperatively. One year after surgery, OCT scans $(\mathbf{b}, \mathbf{d}, \mathbf{f}, \mathbf{h})$ showed resolution of intraretinal fluid, retinal corrugation, and restoration of the ELM, except in 1 eye (h).

\section{Patients and Methods}

This retrospective study was conducted in the Ophthalmology Department of the Hotel-Dieu Hospital in Paris from November 2013 to November 2014.

All consecutive patients presenting with rhegmatogenous RD who were operated on by a single surgeon (M.L.) during this period were included. Exclusion criteria were media opacities, prior vitrectomy, recurrent $\mathrm{RD}$ (i.e., previous surgery for $\mathrm{RD}$ in the study eye), another cause of RD (i.e., exudative or tractional RD), and any history of macular disease.

Collected demographic data included age, gender, and duration of symptoms. All patients underwent a thorough ophthalmologic examination preoperatively and 1 year postoperatively, including a best-corrected visual acuity (BCVA) measurement on a Snellen chart, lens status evaluation, and dilated fundus examination. All recorded Snellen VAs were converted to logarithm of the minimum angle of resolution ( $\left.\log _{\mathrm{MAR}}\right)$ units for statistical analysis. 
Table 1. Description of the study sample at baseline

\begin{tabular}{|c|c|c|c|c|}
\hline & Macula-On & Macula-On/Off & Macula-Off & $p$ value \\
\hline Number of eyes & $25(29.4)$ & $8(9.4)$ & $52(61.2)$ & \\
\hline Female sex & $12(48.0)$ & $4(50.0)$ & $21(40.4)$ & 0.73 \\
\hline Age, years & $57.4 \pm 13.5$ & $55.1 \pm 19.9$ & $61.6 \pm 12.2$ & 0.62 \\
\hline BCVA, $\log _{\text {MAR }}$ & $0.2 \pm 0.2$ & $0.8 \pm 0.5$ & $2.3 \pm 0.8$ & $<0.0001$ \\
\hline BCVA of fellow eyes, $\log _{\mathrm{MAR}}$ & $0.1 \pm 0.1$ & $0.1 \pm 0.1$ & $0.1 \pm 0.2$ & 0.40 \\
\hline Phakic lens status & $22(88.0)$ & $6(75.0)$ & $35(67.3)$ & 0.90 \\
\hline Refraction (spherical equivalent), diopters & $-2.9 \pm 2.5$ & $-1.8 \pm 2.6$ & $-4.7 \pm 5.5$ & 0.40 \\
\hline \multicolumn{5}{|l|}{ RD location } \\
\hline Superior or superotemporal & 16 & 2 & 15 & \\
\hline Inferior or inferotemporal & 4 & 5 & 10 & \\
\hline Strictly temporal & 3 & 0 & 7 & \\
\hline Strictly nasal & 2 & 0 & 0 & \\
\hline Subtotal & 0 & 2 & 11 & \\
\hline Total & 0 & 0 & 9 & \\
\hline Number of clock hours involved & $3.4 \pm 1.2$ & $5.9 \pm 1.8$ & $7.4 \pm 2.9$ & $<0.0001$ \\
\hline Number of tears & $2.0 \pm 1.2$ & $2.3 \pm 1.9$ & $2.2 \pm 1.5$ & 0.96 \\
\hline Symptom duration, days & $0.7 \pm 3.0$ & $11.6 \pm 9.8$ & $14.4 \pm 24.4$ & $<0.0001$ \\
\hline Time to surgery, days & $2.0 \pm 2.1$ & $2.2 \pm 2.0$ & $3.0 \pm .5$ & 0.67 \\
\hline \multicolumn{5}{|l|}{ Surgical procedure } \\
\hline Vitrectomy & $21(84)$ & $6(75)$ & $50(96)$ & \\
\hline Scleral buckling & $4(16)$ & $2(25)$ & $2(4)$ & \\
\hline
\end{tabular}

Values are expressed as mean \pm SD or $n(\%)$. BCVA, best-corrected visual acuity; $\log _{\mathrm{MAR}}$, logarithm of the minimum angle of resolution; $\mathrm{RD}$, retinal detachment.

All patients underwent a noncontact slit-lamp biomicroscopic examination, using a Volk Superfield lens and conducted by 2 retina specialists (M.L. and V.M.), to describe the location, record the extent of detachment in clock hours and the number of breaks, and assess the macular status. On fundus examination, the foveal center was identified as the center of the xanthophyll pigment. Based on this, the included patients were subsequently classified as macula-On RD if the fovea was attached, or macula-Off if the fovea was detached. Patients who were diagnosed with foveal-splitting RD were classified as "macula-On/Off" RD and underwent macular OCT scanning.

Macular OCT was performed on the same day using the Spectralis spectral-domain OCT (Spectralis, Heidelberg Engineering, Heidelberg, Germany). The OCT imaging protocol included 12 linear horizontal scans of 100 averaged images, and 2 single high-definition lines ( 1 vertical and 1 horizontal) centered on the foveal center. The foveal center was identified on OCT as the center of the retinal fixation point of the patient, or the lowest point in the foveal depression. The greatest distance from the foveal center to the RD border and the RD height at the foveal center were manually measured using the caliper function mode of the Heidelberg device (Fig. 1). The presence of intraretinal fluid, retinal corrugation, and ellipsoid-zone and external limiting membrane (ELM) disruption were also recorded. Two investigators (M.L. and V.M.) independently assessed the OCT findings.

Vitreoretinal surgery was performed by a single trained surgeon (M.L.), and the technique used was left to the surgeon's dis- cretion. The ab interno procedure was based on 25-gauge vitrectomy with cryotherapy or laser therapy, and gas or silicon oil tamponade as deemed necessary. Scleral buckling was performed using silicone sponges, and subretinal fluid puncture was performed if needed. Patients were followed up for 1 year after the surgery, and VA measurement and OCT examination were repeated at the last visit.

Quantitative data were reported as mean \pm standard deviation, or median and range, and qualitative values were presented as $n$ (\%). The Kruskal-Wallis test with Dunn's multiple comparisons and the Pearson correlation test were performed when appropriate. $p<0.05$ was considered statistically significant. All statistical analyses were performed using XLSTAT software (Assinsoft, Paris, France).

\section{Results}

Eighty-five consecutive eyes (85 patients) with RD had surgery over the study period and meet the inclusion criteria. Patients' baseline characteristics and clinical data are reported in Table 1. Eight eyes (9.4\%) were diagnosed with foveal-splitting RD by clinical examination. In all 8 of these, the fovea was found to be completely detached on OCT examination. The median distance 
Table 2. Pre- and postoperative visual acuities

\begin{tabular}{lccc}
\hline & Macula-On & Macula-On/Off & Macula-Off \\
\hline Number of eyes & 25 & 8 & 52 \\
Preoperative BCVA, $\log _{\text {MAR }}$ & $0.2 \pm 0.2$ & $0.9 \pm 0.5$ & $2.3 \pm 0.8$ \\
$\quad$ Snellen equivalent & $20 / 30$ & $20 / 160$ & $20 / 4,000$ \\
Postoperative BCVA, $\log _{\text {MAR }}$ & $0.2 \pm 0.1$ & $0.3 \pm 0.3$ & $0.7 \pm 0.7$ \\
Snellen equivalent & $20 / 30$ & $20 / 40$ & $20 / 100$ \\
\hline
\end{tabular}

BCVA, best-corrected visual acuity; $\log _{\mathrm{MAR}}$, logarithm of the minimum angle of resolution.

from the RD border to the foveal center was $632 \mu \mathrm{m}$ (390-2,505 $\mu \mathrm{m})$. This distance was not correlated with the location of the detachment $(1,043 \mu \mathrm{m}$ vs. $844 \mu \mathrm{m}$ for superior and inferior RDs, respectively, $p=0.29)$. The detachment had a median height at the foveal center of $366 \mu \mathrm{m}(100-650 \mu \mathrm{m})$. In 2 cases, the distance between the foveal center and the RD border was $>1,000 \mu \mathrm{m}$ $(2,502$ and $1,206 \mu \mathrm{m}$, respectively), with flat retinal detachment (a height of 418 and $134 \mu \mathrm{m}$ at the foveal center, respectively) (Fig. 1). Preoperative OCT examination showed the presence of intraretinal fluid in 5/8 (62.5\%) eyes, retinal corrugation in 4/8 (50\%) eyes, ellipsoid zone disruption in 4/8 (50\%) eyes, and ELM disruption in 4/8 (50\%) eyes.

The mean baseline BCVA in macula-On/Off eyes was $0.9 \pm 0.5 \log _{\mathrm{MAR}}$, and significantly lower than that measured in the macula-On eyes, but significantly better than that measured in the macula-Off eyes ( $p=0.032$ and $p=0.004$, respectively) (Table 2 ). In the maculaOn/Off eyes, baseline BCVA correlated with RD height at the foveal center $(p=0.04)$, but not with the distance from the foveal center to the RD border $(p=0.87)$. $\mathrm{RD}$ was successfully repaired by a single operation in all macula-On and macula-On/Off eyes, while 2/52 macula-Off eyes required additional operations. No intra- or postoperative complications were noted. In the maculaOn/Off eyes, BCVA improved significantly from $0.9 \pm$ $0.5 \log _{\text {MAR }}(20 / 160$ Snellen equivalent $)$ to $0.3 \pm 0.3 \log$ MAR (20/40 Snellen equivalent) at the 1 -year postoperative examination $(p=0,035)$. One year after surgery, the VA of the macula-On/Off eyes no longer differed from that measured in the macula-On eyes $(p=0.360)$, and tended to be better than that of the macula-Off eyes $(p=0.062)$ (Table 2). Ellipsoid-zone disruption was present in $1 / 8$ eyes $(12.5 \%)$, but the ELM was restored in all eyes.

\section{Discussion}

In this study, we found a difference in the assessment of RD macular extent between fundus examination and OCT, in eyes diagnosed with foveal-splitting RD. In these cases, preoperative OCT examination showed a shallow $\mathrm{RD}$ extending beyond the fovea, with a median distance of $632 \mu \mathrm{m}$ from the foveal center.

Previous studies have reported a difference between fundus examination and OCT to diagnose persistent subretinal fluid after surgery [9-12]. During the preoperative assessment of the macular status, only a few studies have evaluated the use of OCT and reported a discrepancy between fundus examination and OCT findings. In a study conducted on 53 eyes with RD, 13 eyes (23.6\%) were first diagnosed with macula-On RD, based on clinical examination, and then reclassified as macula-Off $\mathrm{RD}$ after preoperative OCT which showed the presence of subfoveal fluid [13]. Auger et al. [14] also reported a case of maculaOn $\mathrm{RD}$ reclassified as macula-Off $\mathrm{RD}$ after OCT examination. Thus, preoperative macular OCT examination may be useful to better ascertain the macular status to be able to inform the patient accurately about his visual prognosis and adjust the timing of the surgical intervention accordingly.

Patients with macula-Off RD have a poorer visual prognosis than macula-On $\mathrm{RD}$ patients $[4,6,15]$. In this study, we showed that the baseline VA of macula-On/Off $\mathrm{RD}$ was better than macula-Off eyes and lower than macula-On eyes seen over the same period. Moreover, after 1 year of follow-up, the VA of macula-On/Off eyes was close to that measured in macula-On eyes (a 20/40 and 20/30 Snellen equivalent, respectively). Hence, the preand postoperative findings usually found in eyes with macula-Off RD do not appear to apply to eyes with what we called macula-On/Off $\mathrm{RD}$, i.e., foveal-splitting $\mathrm{RD}$ based on the fundus examination, but macula-Off based 
on the OCT examination. When RD occurs, loss of vision appears as soon as the foveal center is detached due to rapid degeneration of the photoreceptors [16], and a negative correlation with the detachment height has been reported [17]. The shallow detachment we found in eyes with macula-On/Off RD could explain the intermediate baseline VA. While ellipsoid-zone and ELM disruption were found in half of the eyes at baseline, at the last follow-up examination, only 1 eye still had ellipsoid-zone disruption and no eyes presented with ELM disruption. The low height of the macular detachment, the moderate preoperative visual loss, and the short duration of the detachment could explain the restoration of the ellipsoid zone and ELM and subsequent favorable functional outcomes in this specific group of $\mathrm{RD}[4,6,15,18-20]$. Also, the rate of retinal reattachment after 1 operation was relatively high overall, with only 2 of 85 eyes requiring additional surgery [21-23].

This study has some limitations, including the small sample of macula-On/Off eyes. Fundus examination was not performed with a contact lens. Contact lens examination is more accurate than non-contact examination for the diagnosis of macular disease [24], but a non-contact lens was chosen in this study because it is more often used in clinical practice.

In conclusion, preoperative OCT examination showed a shallow macular detachment extended beyond the fovea in eyes diagnosed with foveal-splitting RD. While these eyes might be considered as macula-Off, their visual prognosis is closer to macula-On eyes. Therefore, prompt surgery should be advised for these cases that we called macula-On/Off RD.

\section{Statement of Ethics}

This study was conducted in a manner compliant with the tenets of the Declaration of Helsinki. The internal review board of Hotel-Dieu Hospital gave a favorable opinion for the conduct of this study and each patient provided oral informed consent as required locally.

\section{Conflict of Interest Statement}

The authors have no conflicts of interest to declare.

\section{Funding Sources}

There was no funding.

\section{Author Contributions}

V.M., M.L., and R.T. conceived and designed the analysis and wrote the paper. V.M. and M.L. collected the data. V.M., M.L., E.B., and P.R.R. performed the analysis. M.L., E.B., P.R.R., R.T, I.C., and E.P. reviewed the paper.

\section{References}

1 Ho SF, Fitt A, Frimpong-Ansah K, Benson MT. The management of primary rhegmatogenous retinal detachment not involving the fovea. Eye (Lond). 2006 Sep;20(9):1049-53.

2 American Academy of Ophthalmology. The repair of rhegmatogenous retinal detachments. Ophthalmology. 1996 Aug; 103(8): 1313-24.

3 Kaufman PL. Prognosis of primary rhegmatogenous retinal detachments. 1. Associations between clinical detachment characteristics, subretinal fluid butyrylcholinesterase and visual outcome following scleral buckling procedures. Acta Ophthalmol (Copenh). 1975 Sep;53(4):660-71.

4 Ross WH. Visual recovery after macula-off retinal detachment. Eye (Lond). 2002 Jul; 16(4):440-6.

5 Kontos A, Williamson TH. Rate and risk factors for the conversion of fovea-on to foveaoff rhegmatogenous retinal detachment while awaiting surgery. Br J Ophthalmol. 2017 Aug; 101(8):1011-5
6 Ross WH, Kozy DW. Visual recovery in macula-off rhegmatogenous retinal detachments. Ophthalmology. 1998 Nov;105(11):2149-53.

7 Fercher AF, Hitzenberger CK, Drexler W, Kamp G, Sattmann H. In vivo optical coherence tomography. Am J Ophthalmol. 1993 Jul;116(1):113-4.

8 Swanson EA, Izatt JA, Hee MR, Huang D, Lin $\mathrm{CP}$, Schuman JS, et al. In vivo retinal imaging by optical coherence tomography. Opt Lett. 1993 Nov;18(21):1864-6.

9 Hagimura N, Iida T, Suto K, Kishi S. Persistent foveal retinal detachment after successful rhegmatogenous retinal detachment surgery. Am J Ophthalmol. 2002 Apr;133(4):516-20.

10 Seo JH, Woo SJ, Park KH, Yu YS, Chung H. Influence of persistent submacular fluid on visual outcome after successful scleral buckle surgery for macula-off retinal detachment. Am J Ophthalmol. 2008 May;145(5): $915-22$
11 Kim YK, Ahn J, Woo SJ, Hwang DJ, Park KH. Multiple subretinal fluid blebs after successful retinal detachment surgery: incidence, risk factors, and presumed pathophysiology. Am J Ophthalmol. 2014 Apr; 157(4):834-41.

12 Benson SE, Schlottmann PG, Bunce C, Xing W, Charteris DG. Optical coherence tomography analysis of the macula after scleral buckle surgery for retinal detachment. Ophthalmology. 2007 Jan;114(1):108-12.

13 Ricker LJ, Noordzij LJ, Goezinne F, Cals DW, Berendschot TT, Liem AT, et al. Persistent subfoveal fluid and increased preoperative foveal thickness impair visual outcome after macula-off retinal detachment repair. Retina. 2011 Sep;31(8):1505-12.

14 Auger G, Winder S. Spectral Domain OCT: An Aid to Diagnosis and Surgical Planning of Retinal Detachments. J Ophthalmol. 2011; 2011:725362. 
15 Burton TC. Recovery of visual acuity after retinal detachment involving the macula. Trans Am Ophthalmol Soc. 1982;80:475-97.

16 Mervin K, Valter K, Maslim J, Lewis G, Fisher $\mathrm{S}$, Stone J. Limiting photoreceptor death and deconstruction during experimental retinal detachment: the value of oxygen supplementation. Am J Ophthalmol. 1999 Aug;128(2): $155-64$.

17 Mowatt L, Tarin S, Nair RG, Menon J, Price NJ. Correlation of visual recovery with macular height in macula-off retinal detachments. Eye (Lond). 2010 Feb;24(2):323-7.

18 Tani P, Robertson DM, Langworthy A. Prognosis for central vision and anatomic reattachment in rhegmatogenous retinal detachment with macula detached. Am J Ophthalmol. 1981 Nov;92(5):611-20.
19 Doyle E, Herbert EN, Bunce C, Williamson $\mathrm{TH}$, Laidlaw DA. How effective is macula-off retinal detachment surgery. Might good outcome be predicted? Eye (Lond). 2007 Apr; 21(4):534-40.

20 Abouzeid $\mathrm{H}$, Wolfensberger TJ. Macular recovery after retinal detachment. Acta Ophthalmol Scand. 2006 Oct;84(5):597-605.

21 Wickham L, Connor M, Aylward GW. Vitrectomy and gas for inferior break retinal detachments: are the results comparable to vitrectomy, gas, and scleral buckle? Br J Ophthalmol. 2004 Nov;88(11):1376-9.
22 Weichel ED, Martidis A, Fineman MS, McNamara JA, Park CH, Vander JF, et al. Pars plana vitrectomy versus combined pars plana vitrectomy-scleral buckle for primary repair of pseudophakic retinal detachment. Ophthalmology. 2006 Nov;113(11):2033-40.

23 Haugstad M, Moosmayer S, Bragadóttir R. Primary rhegmatogenous retinal detachment - surgical methods and anatomical outcome. Acta Ophthalmol. 2017 May;95(3): 247-51.

24 Brun S, Bressler SB, Maguire M, Heiner C, Bressler NM, Schachat AP. A comparison of fundus biomicroscopy and 90 diopter lens examination in the detection of diabetic clinically significant macular edema [ARVO Abstract]. Invest Ophthalmol Vis Sci. 1993;34: 718 